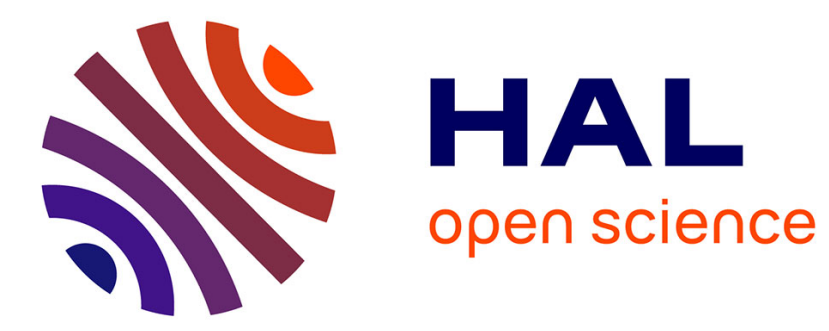

\title{
Les censeurs, premiers critiques de bande dessinée
}

Sylvain Lesage

\section{To cite this version:}

Sylvain Lesage. Les censeurs, premiers critiques de bande dessinée. Revue de la Bibliothèque nationale de France, 2020, Ne les laissez pas lire! Censure dans les livres pour enfants, 60. hal-02570763

\section{HAL Id: hal-02570763 \\ https://hal.science/hal-02570763}

Submitted on 12 May 2020

HAL is a multi-disciplinary open access archive for the deposit and dissemination of scientific research documents, whether they are published or not. The documents may come from teaching and research institutions in France or abroad, or from public or private research centers.
L'archive ouverte pluridisciplinaire HAL, est destinée au dépôt et à la diffusion de documents scientifiques de niveau recherche, publiés ou non, émanant des établissements d'enseignement et de recherche français ou étrangers, des laboratoires publics ou privés. 


\title{
Les censeurs, premiers critiques de bande dessinée
}

\author{
Sylvain Lesage
}

Des années 1920 aux années 1970, la bande dessinée, médium privilégié de la culture enfantine, suscite une méfiance unanime, que ne fait que renforcer la loi de 1949 sur les publications destinées à la jeunesse. Pour mieux lutter contre ces récits en images jugés immoraux les censeurs seront les premiers à les étudier et les définir, se transformant en véritables critiques littéraires...

Dans Contre la bande dessinée (L'Association, 2008), l'artiste Jochen Gerner recense et illustre les poncifs qui contribuent à la mauvaise réputation des "petits mickeys »; il esquisse, par ce biais, un panorama des clichés contre lesquels auteurs et amateurs ont dû lutter pour faire reconnaître la dignité du neuvième art. Gerner attire l'attention sur un point crucial : les décennies de discours alarmistes et d'écrits effrayés par les effets délétères de la bande dessinée n'ont pas seulement contribué à dévaloriser les littératures en images. Les censeurs divers qui, dès les années 1920, s'échinent à voir dans la bande dessinée le symptôme d'une décadence culturelle sont aussi - non sans une once de paradoxe- les premiers à l'avoir théorisée.

Dans le domaine de la littérature, du théâtre ou du cinéma, de nombreux travaux ont mis en relief l'importance des censeurs dans les réflexions théoriques et stylistiques. Jean-Baptiste Amadieu a ainsi souligné combien la réalisation de l'Index librorum prohibitorum repose sur une évaluation stylistique, au point de transformer les censeurs en véritables critiques littéraires i. Frédéric Hervé a, quant à lui, montré pourquoi, dans un tout autre contexte, les films français des «trente glorieuses» sont à la fois "les enfants du Cinématographe et

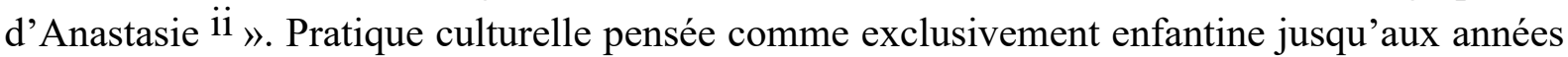
1960-1970, la bande dessinée constitue ainsi pendant longtemps un loisir sous surveillance. Si, comme Thierry Crépin l'a écrit, les effets de la loi de 1949 sur les publications destinées à la jeunesse reposent davantage sur l'autocensure que sur une intervention directe de la commission chargée de sa mise en œuvre, ses effets n'en sont pas moins réels. Pour complaire aux desiderata des commissaires et éviter l'arbitraire de sanctions administratives impitoyables, éditeurs et rédacteurs relaient des consignes narratives et stylistiques qui forgent les caractéristiques de la bande dessinée "franco-belge » de l'après-guerre : conformisme moral, femmes reléguées à l'arrière-plan et désexualisées, rejet des genres de l'imaginaire telles en sont quelques-unes des caractéristiques les plus visibles. Mais les conséquences de cette sourcilleuse vigilance exercée à l'encontre de la bande dessinée dépassent de loin la seule question de la chape de plomb qui pèse sur la création.

En effet, avant l'émergence, dans les années 1960, des premiers cercles bédéphiles, les seuls travaux qui se penchent sur la bande dessinée sont le fruit de cette méfiance vis-à-vis des narrations en images. Pourtant, la réflexion théorique sur la bande dessinée est sans doute aussi ancienne que la bande dessinée elle-même: les intuitions du pédagogue genevois Rodolphe Töpffer, rassemblées dans son Essai de physiognomonie, restent éclairantes encore aujourd'hui : Töpffer élabore la première analyse théorique du médium, en même temps qu'il en élabore la grammaire formelle. Mais après Töpffer, la réflexion théorique sui generis disparaît corps et biens; cousine de la caricature, la bande dessinée n'est pas perçue comme une pratique distincte, et elle n'attire guère l'attention des élites culturelles. C'est lorsqu'elle entame sa mue pour devenir, au tournant du $\mathrm{XX}^{\mathrm{e}}$ siècle, la forme privilégiée d'une culture enfantine passant par les journaux illustrés qu'elle commence à susciter l'attention. Ainsi, pendant plusieurs décennies, les censeurs ont été les seuls à proposer une réflexion sur la bande dessinée, son langage, ses modes de production, sa réception, ses lecteurs... Elle est 
alors vue à travers des prismes très particuliers : le combat pour les bonnes lectures de militants catholiques effrayés par l'irréligion de leurs contemporains, le combat de militants de la lecture publique contre les ravages de la culture de masse, l'atmosphère de panique morale des années 1940, la guerre froide culturelle, etc.

La production critique de ces censeurs est vaste et diversifiée: brochures, pamphlets, recensions, circulaires... Elle révèle, avec des nuances importantes d'un(e) auteur(e) à l'autre, une ambition de comprendre la bande dessinée afin de mieux lutter contre elle, fournissant ainsi le premier cadre théorique que les bédéphiles des années 1960 et les chercheurs qui leur emboîtent le pas vont entreprendre de déconstruire.

\section{Comprendre la bande dessinée}

La première difficulté qu'affrontent ces censeurs est, paradoxalement, de penser la bande dessinée; la désignation même du médium pose problème. Dans les années 1930, on parle indifféremment d' " illustrés », de « séries illustrées », d' " histoires en images », de " dessins accompagnés de légendes »... S'il est délicat de dater précisément l'apparition du syntagme «bande dessinée », une chose paraît certaine : les débuts de sa banalisation auprès du grand public coïncident avec l'adoption d'une législation restrictive sur les publications destinées à la jeunesse. Si le terme n'apparaît pas spécifiquement dans les débats parlementaires, il se répand dès le début des années 1950.

Les flottements sémantiques traduisent, plus largement, une difficulté à appréhender les spécificités sémiotiques du médium, et la délicate articulation texte-image qui porte la narration. Ainsi, lorsque la bulle de dialogue se généralise, dans les années 1930, l'incompréhension des bibliothécaires de l'Heure joyeuse est patente dans les fiches bibliographiques qu'elles rédigent pour la rédaction de Beaux livres, belles histoires: Jacqueline Dreyfus-Weil note à propos de Zig et Puce aux Indes, d'Alain Saint-Ogan, «Présentation détestable: les textes sortent de la bouche des personnages iii ». Cette incompréhension est partagée par Marguerite Gruny, qui écrit par exemple à propos du Génial M. Poche, d'Alain Saint-Ogan toujours : "Album d'images : plusieurs petites images par page formant une historiette comique, dialoguée (les paroles des personnages sont entourées comme sortant de leurs bouches) iv. " L'hostilité que vouent les bibliothécaires à la bande dessinée se traduit donc par un inconfort, et une difficulté à penser l'articulation texte-image comme l'une des spécificités de la bande dessinée. Deux décennies plus tard, l'incompréhension des éducateurs reste d'actualité, par exemple dans l'article consacré à la presse pour enfants en France par Simone Lacroix dans le Bulletin des bibliothèques de France en 1956 :

La stylisation du dessin, qui schématise les personnages et le milieu, les réduisant à quelques éléments essentiels mais leur donnant aussi plus de relief, facilite la compréhension de l'histoire par une vision rapide et continue. Il reste que ces options diverses de l'illustrateur donnent l'impression d'un temps discontinu dont souffre la logique interne du récit. D'où ces « ballonnets » sortis de la bouche des personnages ne renfermant que quelques lignes et dont le rôle est d'assurer la liaison avec l'image suivante, chevilles entre deux images.

$\mathrm{Si}$, rétrospectivement, cette difficulté à saisir les mécanismes sémiotiques qui guident la lecture de bande dessinée peut faire sourire, elle témoigne de la progressive élaboration d'un langage analytique pour décrypter le fonctionnement des récits en images.

\section{Un idéal de lisibilité}

Mais, au-delà de cette difficulté à cerner le langage de la bande dessinée, les censeurs font œuvre de critiques. Rares en effet sont les éducateurs ou les censeurs à condamner tous les illustrés; la plupart du temps, ils entreprennent de classer, de préconiser des substituts acceptables, voire de les produire eux-mêmes. Leur démarche repose donc fondamentalement 
sur une évaluation, qui fait de ces censeurs les premiers critiques de bande dessinée - quand bien même ils se livrent à cette activité de façon férocement engagée.

Les bibliothécaires de l'Heure joyeuse sont symptomatiques de ce mouvement. Ouverte en 1924 grâce à des fonds américains destinés à la reconstruction, la bibliothèque tranche par son public de destination (les enfants de cinq à seize ans) et par le libre accès aux ouvrages. Prototype de la bibliothèque moderne, l'Heure joyeuse est animée par trois bibliothécaires qui réalisent un travail considérable de critique, de sélection et de prescription, largement diffusé auprès du réseau d'apprenties passées par les locaux de la rue Boutebrie. Les bibliothécaires de l'Heure joyeuse forment ainsi un groupe de témoins intéressants, car leur aversion vis-à-vis de la bande dessinée n'empêche pas des accommodements avec les demandes des enfants ; mais leur méfiance les pousse à des examens extrêmement stricts de la production éditoriale, et elles sont particulièrement attentives aux qualités formelles et à la lisibilité des albums (l'illustré, lui, n'a pas droit de cité). Ainsi, Frimousset au collège, de Jaboune et Pinchon (1924), s'attire ce jugement lapidaire : « Aucune valeur artistique ni surtout littéraire. Banalité morne. Texte mélangé aux illustrations sans ordre - dans tous les sens - aspect de journal illustré bon marché. » La composition très libre de Pinchon se voit ainsi condamnée en raison des obstacles qu'elle ferait peser sur la compréhension du récit. Le reproche le plus récurrent concerne cependant l'impression du texte. Dans Grassouillet pêche à la ligne (1928), Mathilde Leriche condamne une " impression trop fine»; en revanche, en 1955, Le Temple $d u$ Soleil reçoit un satisfecit concernant sa « typographie très nette » $(\mathrm{sic})$.

Cette attention portée aux caractéristiques formelles n'est pas l'apanage des bibliothécaires. On trouve en fait sous la plume de presque tous les censeurs une attention soutenue aux qualités stylistiques. Ainsi, la célèbre brochure produite par le Cartel d'action morale et sociale en 1944 ne fait pas exception :

Ces journaux concourent au nivellement de la jeunesse par le médiocre. Ainsi le héros, rendu populaire par ces journaux, est Tarzan, demi-sauvage, qui tient plus de la bête que de l'homme. Nivellement aussi par la laideur : la présentation est laide, les illustrations sont affreuses, nulle poésie dans le texte ${ }^{\mathrm{V}}$.

La brochure intitulée La Démoralisation de la jeunesse par les publications périodiques explique le "succès foudroyant de la presse malsaine » par "l'attrait - bien dangereux d'histoires troubles [...] et d'images excitantes $\mathrm{vi} »$. Il est frappant de relever que, cherchant à décrire le caractère dangereux de cette littérature enfantine, les auteurs répondent notamment à travers la question du style. L'évaluation stylistique s'y fait certes sommaire; pour être péremptoire, elle n'en est pas moins centrale dans le rejet et la dénonciation d'une bande dessinée « étrangère » perçue comme agitée et brouillonne, car mettant en œuvre des codes esthétiques bien éloignés du Christophe des années 1880, qui représente le versant acceptable des récits en images.

En juin 1939, la revue Le Patronage, éditée par Fleurus, consacre un long article au " problème » de la presse illustrée pour enfants. Parmi les attaques dirigées largement contre les publications Winkler et Del Duca, on retrouve là aussi l'embryon d'une condamnation stylistique de la bande dessinée :

Si nous prenons, au hasard, les illustrés parus dans l'une de ces dernières semaines, nous nous apercevons que [l'essentiel] ne semble avoir qu'un seul but : abêtir les enfants par des histoires incompréhensibles, idiotes, dénuées de bon sens, ou par des récits d'aventures invraisemblables, effrayantes, scabreuses; tandis que leur jeune imagination est nourrie d'images déformatrices du vrai ou du beau, aux expressions aussi peu éducatives que possible et d'une tenue parfois, souvent même, fort peu correcte vii.

La posture de dénonciation de la bande dessinée dans l'entre-deux-guerres et largement prolongée par la suite, portée par les figures de la moralisation de la jeunesse, mêle étroitement laideur esthétique et perte du réel. À ce titre, une corrélation étroite est établie entre d'un côté la qualité de la syntaxe et de l'orthographe, la vivacité des couleurs, le trait, et de l'autre une décadence morale : à la décadence des mœurs correspondrait une décadence du dessin. 


\section{La question du style}

Lors des débats au Conseil de la République autour de l'adoption de la loi de 1949, le conseiller socialiste Pierre Pujol justifie ainsi l'adoption d'une législation restrictive concernant l'imprimé pour enfants : «On a fait le procès du cinéma et du journal illustré ; le cinéma a cependant cet avantage, sur le plan moral, que, la séance finie, l'image s'évanouit peu à peu de la mémoire, tandis que l'image, dans le journal illustré, étalé au kiosque, acheté, reste, persiste, peut être découpée, conservée par l'enfant, l'image du gangster au corps épais, à la tête minuscule, imposant aux mœurs la dictature de la mitrailleuse viii. »

On retrouve cette attention portée aux éléments stylistiques dans la surveillance sourcilleuse qu'exerce la commission chargée de la mise en œuvre de la loi de 1949 sur les publications destinées à la jeunesse. Alors que les écarts établis par la loi sont uniquement d'ordre moral (la prohibition de la présentation "sous un jour favorable [du] banditisme, [du] vol, [de] la paresse », etc.), les commissaires s'emploient bien vite à tenter de remodeler le contenu des publications destinées à la jeunesse. Ainsi, dans son rapport de 1955, la commission reproche « l'habitude trop généralement suivie d'abuser des bandes dessinées qui remplissent presque la publication : le texte écrit se trouve alors réduit à des "ballons" et à des onomatopées ix 》. Les commissaires rejettent un certain style graphique perçu comme étranger, et particulièrement américain, malgré plus de deux décennies d'usage dans la presse française. De même, les commissaires enjoignent aux éditeurs de soigner la présentation :

Il arrive trop souvent que [...] l'impression présente les pires défectuosités (encore plus sensibles quand les caractères sont trop petits) ou que l'aspect d'ensemble soit pénible à la vue ; comme c'est le cas lorsque des couleurs violentes et heurtées s'appliquent sur des dessins imprimant à la réalité d'excessives déformations $\mathrm{x}$.

Loin de se limiter à des considérations générales, les commissaires se livrent parfois à une critique attentive des publications qui passent entre leurs mains; ainsi, lorsqu'une souscommission «Artima » est créée, en 1960, le récit "L’Épée brisée », publié dans le bimensuel Oliver, fait l'objet de la remarque suivante : "Dessin mauvais. Têtes laides. La trame du récit reste obscure. Image d'un bateau sarrazin [sic] de la plus haute fantaisie. »

Ces condamnations sur motifs stylistiques se retrouvent jusqu'à la fin des années 1980 tout au long des séances de la Commission de surveillance - alors même que la commission perd graduellement son pouvoir de nuisance. Lors de la séance du 16 avril 1975, au cours de l'examen d'une vingtaine de titres de l'éditeur Elvifrance, Raoul Dubois fait état du « succès grandissant de ces revues à l'intérieur des établissements scolaires, et en particulier dans les classes de $6^{\mathrm{e}} \gg$; il ajoute qu'il s'agit

de publications extrêmement nocives, d'une part parce qu'elles utilisent un graphisme agressif, d'autre part à cause de la violence de leur vocabulaire. Ce genre de publications n'a pas l'excuse d'une quelconque qualité esthétique. Aussi, la Commission se doit d'être très sévère dans sa décision. Il lui faut en particulier protéger les enfants et les adolescents de la laideur qui peut résulter de la présentation de ces revues aux étalages de presse $\mathrm{xi}$.

De même, quatre ans plus tôt, le secrétaire de la commission va jusqu'à convoquer Georges Rieu, à l'époque rédacteur en chef de Pif, pour le morigéner. La publication de Corto Maltese fait l'objet de vives réprimandes en raison des «violences répétées du récit [...] encore renforcées par un dessin très noir». Il est en particulier reproché au récit d'Hugo Pratt «le nombre et la dimension des canons braqués vers le lecteur, sur trois pages successives, le style agressif de ces images, ainsi que les onomatopées ponctuant des péripéties très agitées ». Malgré leur absence de bagage théorique, les commissaires s'improvisent donc censeurs des élégances stylistiques, outrepassant les limites assignées à leur mission de moralisation de la jeunesse.

Des années 1920 au tournant éducatif xii des années 1970, le consensus est large auprès de la communauté éducative pour stigmatiser les effets délétères de la bande dessinée. Au tournant des années 1970, de nouvelles menaces culturelles prennent le dessus: les mangas qui apparaissent à la télévision française, les jeux vidéo... La bande dessinée perd alors une bonne partie de son prétendu pouvoir de nuisance, et les discours catastrophistes se reportent 
alors, souvent à l'identique, sur ces nouveaux venus des cultures enfantines. Entre-temps, les discours des censeurs ont maintenu un éteignoir sur la production locale et ont tâché d'endiguer les productions perçues comme non conformes à un esprit français ; Émile Keirsbilk, le gérant de l'éditeur tourquennois Artima, en fera abondamment les frais.

En se lançant en pionniers dans la critique de bande dessinée, les censeurs contribuent donc, non sans paradoxe, à poser les bases de son étude, qui seront reprises par les bédéphiles des années 1960 et 1970. Car ceux-ci devront d'abord s'échiner à déconstruire des décennies de discours dénonçant les effets néfastes de la bande dessinée. La tonalité apologétique qui imprègne alors les discours de la première génération de chercheurs sur la bande dessinée doit donc beaucoup aux efforts des censeurs pour disqualifier les littératures en images.

\footnotetext{
' Jean-Baptiste Amadieu, Le Censeur critique littéraire. Les Jugements de l' "Index », du romantisme au naturalisme, Paris, Hermann, 2019, 632 p.

ii Frédéric Hervé, Censure et cinéma dans la France des «trente glorieuses », Paris, Nouveau Monde, 2015, p. 26.

iii Archives de l'Heure joyeuse, fiches bibliographiques de Jacqueline Dreyfus-Weil.

iv Archives de l'Heure joyeuse, fiches bibliographiques de Marguerite Gruny.

v Daniel Parker et Claude Renaudy, La Démoralisation de la jeunesse par les publications périodiques, Paris, Cartel d'action morale et sociale, 1944, p. 11.

vi Ibid., p. 9.

vii Paul-Émile Grosse, « Les illustrés pour enfants », Le Patronage, juin 1939, p. 7.

viii Pierre Pujol, J.O.R.F., Débats parlementaires, Conseil de la République, 5 mars 1949, 19éance, p. 534.

ix Archives nationales, CSC, 19900208, art. 1, Compte rendu des travaux de la Commission de surveillance et de contrôle des publications destinées à l'enfance et à l'adolescence, 1955.

x Ibid.

xi Archives nationales, CSC, 19900208, art. 7, PV de la 104e séance du 16 avril 1975.

xii Antoine Roux, La bande dessinée peut être éducative, Paris, Éditions de l'École, 1970.
} 